\title{
Riesgos laborales para estudiantes, pasantes y practicantes empresariales. Análisis regional ${ }^{1}$ Occupational risks for students, graduate interns and company practitioners. A regional analysis.
}

\author{
LUIS ALBERTO TORRES TARAZONA ${ }^{2}$ \\ albertotota@hotmail.com \\ Mónica Alexandra Cifuentes CruZ, Sandra Milena Corredor Torres \\ LEIDY DAMARIS RUbio MURCIA, LiLIANA MARCELA ZAMBRANO ALDANA ${ }^{3}$
}

RESUMEN

El presente escrito expone el trabajo investigativo que se sustenta en el análisis jurídico del numeral $3^{\circ}$, del literal (a) del artículo 13 del Decreto 1295 de 1994, normativa colombiana que reglamenta la obligatoriedad de la afiliación al Sistema de Riesgos Laborales de los estudiantes, practicantes o pasantes.

150 A partir de un ejercicio de derecho comparado se pretende demostrar la falta de eficacia de la norma y la necesidad de implementar medidas que conlleven su aplicación.

Se abordará, en primer lugar, el concepto de riesgos laborales, a fin de contextualizar al lector y enfocar la investigación realizada; seguidamente se realizará una observación de la regulación adoptada en diferentes naciones y, por último, se hará el estudio de la aplicabilidad de la reforma al Sistema de Riesgos Profesionales (Ley 1562 de 2012), hoy Riesgos Laborales.

PALABRAS CLAVES: riesgos laborales, estudiantes, practicantes, Latinoamérica, derechos, obligatoriedad.

Fecha de recepción: marzo 15 de 2013

Fecha de aceptación: abril 16 de 2013

\section{ABSTRAC}

This research work is based on the analysis of section 3, of paragraph (a) of Article 13 of Decree 1295 of 1994, legislation Colombian regulating compulsory membership system Occupational hazards of students, hence, to from a legal analysis of comparative law is to prove the lack of efficacy and the necessary application.

The document addressed the concept of occupational hazards in order to contextualize the reader and focus research, consecutively, will conduct an observation of the rules adopted in different nations, ending with the study of the applicability of the system reform irrigation Professionals, Law 1562 of 2012, today Safety.

KEYWORDS: occupational hazards, students, practitioners, Latin America, rights, compulsory.

1. Artículo de reflexión resultado del análisis de derecho comparado realizado dentro del proyecto de investigación Prácticas empresariales y pasantías, obligación de afiliación a estudiantes universitarios por parte de los centros académicos, al sistema de riesgos laborales vs. principio utilidad de la norma, en Bogotá, D.C. - periodo 2010-2011, de la Facultad de Derecho de la Universidad Libre.

2. Candidato a magister en Derecho Administrativo; especialista en Derecho Laboral y Seguridad Social; docente de pregrado de las universidades Libre y Autónoma; docente investigador del posgrado de Derecho Laboral y Seguridad Social de la Universidad Libre y coordinador de la investigación que aquí se presenta.

3. Estudiantes de la Facultad de Derecho de la Universidad Libre e investigadoras. 


\section{Introducción}

Es clara la existencia de un problema al no proteger de forma adecuada a los estudiantes que realizan la práctica empresarial o pasantía, en razón a que se ven expuestos a los mismos riesgos que corre un trabajador, pero sin tener esta calidad; consecuentemente, ven vulnerados derechos tales como: la salud, la seguridad social e inclusive la vida (Constitución Política, 1991). De aquí que es el Estado, en calidad de salvaguardia de los derechos mínimos fundamentales del hombre (como los reconocidos por la Constitución y la jurisprudencia constitucional), es quien debe velar por la atención a la salud del estudiante universitario y dar total garantía al cumplimiento de su afiliación obligatoria al Sistema de Riegos Laborales por parte de las universidades, empresas o entidades correspondientes.

Hacia 1991 surge el Estado Social de Derecho, y con él la primacía de los derechos fundamentales sobre la ley, cambio de gran importancia en la legislación, puesto que con este nuevo sistema constitucional nace una forma de interpretación, en la cual estos derechos adquieren rango de aplicación suprema y exigen su efectiva protección por parte del Estado. Así, la seguridad social determinada en el artículo 48 se constituye en un derecho fundamental del cual goza cada ser humano con sujeción a los principios de eficiencia, universalidad y solidaridad, tal y como lo determina la ley suprema.

Como consecuencia de este precepto constitucional el Estado colombiano promulga la Ley 100 del 1993, con la cual se crea el Sistema de Seguridad Social Integral, al que por disposición de esta pertenece el Sistema de Riesgos Profesionales, hoy Riesgos Laborales. En desarrollo de la mencionada ley, el extinto Ministerio de Trabajo y Seguridad Social expidió el Decreto-Ley 1295 de 1994, el cual determina la organización y administración del Sistema General de Riesgos Profesionales y dispone, a su vez, en el literal (a) numeral $3^{\circ}$ del artículo 13, la afiliación “obligatoria” de los estudiantes universitarios, que estén realizando algún trabajo que signifique fuente de ingreso para la respectiva institución donde laboran.

Los avances que ha venido realizando el Estado para el cumplimiento de esta disposición han sido escasos. Aunque en cabeza del Ministerio de Salud se promulga el Decreto 190 de 1996, reglamentario de la relación Docente-Asistencial en el Sistema General de Seguridad Social en Salud, cuyo objetivo es la asistencia dirigida únicamente a estudiantes de pregrado y postgrado en el ámbito de la salud, que realizan sus prácticas prestándole un servicio a la comunidad, este estipula que serán de obligatoria afiliación al Sistema de Riesgos los inscritos en este programa.

Por otra parte, y siguiendo la idea de aproximación del legislador a la cobertura de riesgos a los estudiantes universitarios practicantes, históricamente se protege a aquellos que en su calidad de aprendices prestan un servicio. 
El Decreto-Ley 1295 de 1994 fue modificado por la Ley 1562 del 11 de julio del 2012, la cual cambia la denominación riesgos profesionales por riesgos laborales, y en el artículo $2^{\circ}$ modifica el artículo 13 del Decreto-Ley, disponiendo en el literal (a) numeral $4^{\circ}$ la afiliación obligatoria al Sistema General de Riesgos Laborales "de estudiantes de todos los niveles académicos de instituciones educativas públicas y privadas que deban ejecutar trabajos que signifiquen fuente de ingreso para la respectiva institución o cuyo entrenamiento o actividad formativa es requisito para la culminación de sus estudios”.

Tanto la jurisprudencia como la doctrina colombiana no han desarrollado de manera puntual la problemática expuesta, remitiéndose solo a la mención del artículo, mas no a su tratamiento, de modo que el problema persiste.

En este orden de ideas, han sido pocos los escritos que se han realizado al respecto. Por ello, la presente investigación se ha realizado con el fin de examinar la implementación del artículo que estipula la afiliación obligatoria de los estudiantes universitarios, practicantes y pasantes al Sistema General de Riesgos Laborales por parte de las universidades, instituciones o empresas, en consideración a los riesgos que el trabajo social les representa para su vida, y la protección que se desprende del Estado Social de Derecho.

La investigación responde a la siguiente pregunta: ¿cómo se regula en países latinoamericanos a los estudiantes universitarios en las prácticas empresariales o pasantías, frente a la responsabilidad de afiliación al Sistema de Riesgos Laborales?

De acuerdo con lo anterior, el trabajo que aquí se presenta pretendió demostrar, a manera de hipótesis que en Colombia no está plenamente regulado este aspecto, aunque sí reglamentado, para lo cual se realizó el análisis de derecho comparado entre nuestras disposiciones y las de países latinoamericanos.

\section{Estrategia metodológica}

\section{Enfoque de la investigación en derecho}

El enfoque en derecho de la investigación fue de carácter socio-jurídico, dado que tanto el objeto de estudio como el campo de acción pretenden demostrar que no es suficiente la regulación existente y sus efectos en el mundo material, del mismo modo que en la vida social; de ahí el interés de demostrar la ausencia del principio de utilidad de la norma, en lo que respecta al literal (a) del artículo 13 del Decreto 1295 de 1994, modificado por el artículo $2^{\circ}$ de la Ley 1592 de 2012. 


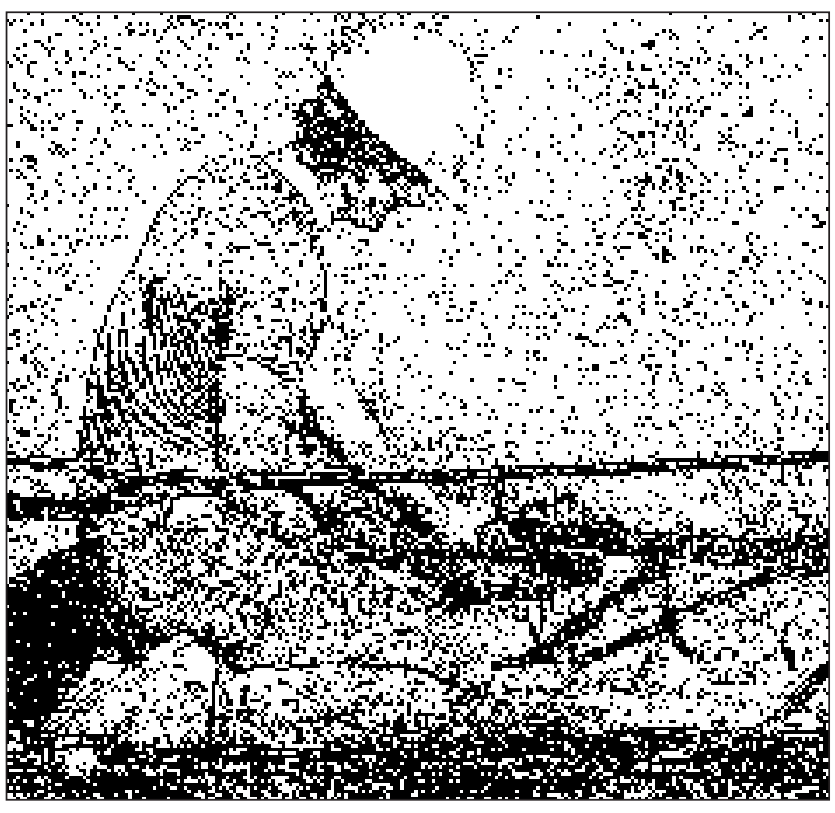

Los riesgos profesionales, laborales u ocupacionales, son daños eventuales generados por el desempeño, actividad u oficio; existen otros riesgos propios del medio ambiente, a los que la actividad laboral añade factores que modifican las condiciones naturales, pero también las sociales y personales, creando una serie de circunstancias que en forma de agentes causales son responsables de muchas enfermedades o accidentes laborales.

En este orden de ideas, existe una población afectada, en este caso los estudiantes universitarios, que dentro de sus prácticas empresariales o pasantías laboran sin recibir alguna protección por parte de los claustros universitarios, respecto al riesgo evidente que representa cualquier tipo de trabajo.

\section{Tipo de investigación}

Es preciso subrayar que este problema investigativo no ha sido abordado con anterioridad en Colombia, por lo cual no existe un estado del arte. Nos encontramos, entonces, ante un estudio novedoso que aportará a posteriores investigaciones que se realicen al respecto, que define la investigación como de carácter, y que avanza en un estado del arte a nivel general.

\section{Método de investigación}

El problema investigativo expuesto fue resuelto a través de dos aspectos metodológicos: uno teórico y el otro práctico o empírico. Al igual que en materia de derecho el método teórico usado fue el Inductivo, puesto que el problema científico se resuelve partiendo de lo singular, es decir, de los hechos, en este caso la falta de afiliación de los estudiantes universitarios al Sistema de Riesgos Laborales durante sus prácticas empresariales o pasantías, para encontrar respuesta en lo general: la seguridad social en Colombia como derecho fundamental, a partir del principio de utilidad de la norma. 
La investigación realizada es de carácter cuali-cuantitativo, debido a que el tema se desprende de una cualificación de los hechos, es decir, de una descripción de tipo valorativo del problema social, como es la falta de afiliación de los estudiantes universitarios al Sistema General de Riegos Laborales, según la Ley 1562 de 2012 durante el tiempo de prácticas, para llegar a un modelo estructurado del cual se desprende una hipótesis en búsqueda de una novedad científica: el principio de utilidad de la norma como posible solución al problema presentado.

\section{Marco histórico del concepto de riesgo profesional, laboral u ocupacional}

Desde la antigüedad el ser humano se ha visto expuesto a diferentes clases de riesgos. Pero, ¿qué se define como riesgo? Según pensadores como Fischhoff, Watson y Hope (1984, 03), la definición consiste en tener en cuenta los valores de la sociedad y determinar sus consecuencias a futuro, de tal manera que estas, combinadas, pueden generar un indicador de riesgo.

Yates y Stone (1992, 04), citan el Diccionario de la Real Academia Española que define riesgo como la "contingencia o proximidad de un daño". Sin embargo, el riesgo no solo se refiere a la contingencia o posibilidad de que algo suceda, sino también a las causas o factores de riesgo como actividades, tecnologías, sustancias o acontecimientos. En este sentido, por ejemplo, fumar, la energía nuclear, una mala posición al realizar una actividad serían un riesgo.

En la sociedad los riesgos son inescindibles, ya que a medida que avanza el desarrollo va generando una serie de peligros, como lo muestra el proceso que arrancó con la revolución industrial y actualmente ha supuesto un cambio radical en el modo de producción y en los procesos que este implica.

En el desarrollo de la sociedad se ve la utilización de instrumentos, máquinas, aparatos, energías, sustancias, que hacen muy compleja la salud y la calidad de vida de las personas que participan en el proceso de producción. Es lo social, la revolución industrial implicó la transformación del trabajo solitario del artesano al trabajo en masa de las fábricas, la separación del trabajador y los medios de producción, y una organización ajena que constituyó el proceso productivo, los métodos de trabajo, las condiciones laborales: ritmos, jornadas y descanso (Puyana, 1995, pp. 5 y 6) y, con ello, factores de riesgo constantes, a cuya exposición se pueden generar alteraciones en la salud física o mental.

Con la aparición de estos nuevos modelos de producción el ser humano se ha tenido que enfrentar a varias transformaciones en las condiciones de vida y de salud, así a los accidentes y enfermedades laborales que trajeron consigo y que dieron lugar a la creación de un sistema de seguridad social que proteja al trabajador de los peligros que se han generado. 
El hombre, en la realización de cualquier tipo de actividad, genera o se expone a diferentes clases de riesgos, entre ellos, los que se desarrollan en virtud de actividades propias de la profesión de cada individuo.

Los riesgos en Colombia se clasifican dependiendo de la actividad que se realiza. Así mismo, cada tipo de riesgo genera unos factores, estos últimos entendidos como todo elemento cuya presencia o modificación aumenta la probabilidad de producir un daño o lesión en quien está expuesto a él (Corporación Ambiental Empresarial, 2006). Las personas, en su trabajo, realizan diversas actividades y dependiendo de cómo las ejecutan se determina la clase y grado de riesgo que enfrentan.

Para valorar el nivel de riesgo que tiene una actividad económica -al interior de las empresas o entidades- se aplica el artículo 26 del Decreto 1295 de 1994 según el cual, se presentan las siguientes clases de riesgos: Clase V Riesgo máximo; como por ejemplo areneras, proceso de asbesto, bomberos, manejo de explosivos, construcción, petroleras.

Clase IV Riesgo alto; por ejemplo procesos manufactureros como aceites, cervezas, vidrios, procesos de galvanización, transporte.

Clase III Riesgo medio; por ejemplo procesos manufactureros como fabricación de agujas, alcoholes, alimentos, automotores y artículos de cuero.

Clase II Riesgo bajo; por ejemplo algunos procesos manufactureros como la fabricación de tapetes confecciones y flores artificiales.

Clase I Riesgo mínimo; por ejemplo la mayor parte de actividades comerciales, actividades financieras, trabajos de oficina, centros educativos, o restaurantes.

(Ministerio de Trabajo y Seguridad Social. Conozca el Sistema General de Riesgos Profesionales. 1995. Universidad Pontificia Javeriana).

Ahora bien, a cada nivel de riesgo le corresponde un valor para el monto de la cotización, la cual esta exclusivamente en cabeza -su pago- del empleador, pero que dependiendo de la modalidad de vinculación, relación o contrato será cancelada ya sea, por el empleador, trabajador independiente, contratista o contratante, puesto que la ley 1562 de 2012 determina estas nuevas reglas.

La clasificación de las actividades económicas que corresponden a cada clase de riesgo está contenida en una amplia lista del Decreto 1607 de 2002. Si la actividad no aparece relacionada en la tabla, el empleador y la ARL podrán efectuar la clasificación de acuerdo con el riesgo ocupacional de la actividad afín contemplada en la tabla.

En principio, entonces, a cada empresa le corresponde exclusivamente una clase de riesgo, sin embargo está previsto que cuando una misma empresa tenga más de un centro de trabajo podrá tener diferentes clases de riesgo, para cada uno de ellos por separado (Arenas Monsalve, 2011, pp. 654-655). 
Los riesgos profesionales, laborales u ocupacionales, son daños eventuales generados por el desempeño, actividad u oficio; existen otros riesgos propios del medio ambiente, a los que la actividad laboral añade factores que modifican las condiciones naturales, pero también las sociales y personales, creando una serie de circunstancias que en forma de agentes causales son responsables de muchas enfermedades o accidentes laborales.

Carlos Posada (1994, p. 19) expresa la existencia de dos tipos de riesgos que "por su origen pueden ser, riesgos fisiológicos y económico-sociales. Estas dos fuentes únicas de posibles riesgos contra las cuales protegen los llamados Seguros Sociales”. Jesús María Rengifo (1989, pp. 71,72) no niega que lo anterior sea una teoría acertada pero busca complementarla de conformidad con la doctrina de Efrén Borrajo Dacruz, quien en su obra Estudios jurídicos de previsión social (1962, p. 145), plantea una clasificación menos complicada y más comprensible para su aplicabilidad en el derecho español, a saber: una primera clasificación referida a riesgos profesionales como son los accidentes de trabajo, la enfermedad profesional y el paro involuntario; la segunda, referida a riesgos no profesionales, divididos en los de naturaleza biológica como enfermedad, maternidad, vejez e invalidez, y los de naturaleza económico-social como obligaciones familiares.

156 En nuestro país existen varios factores de riesgo que la Corporación Ambiental Empresarial (2006), los clasifica de la siguiente manera: físicos, químicos, biológicos, psicosociales, por carga física, mecánico, eléctrico, locativo, físico-químico, y público.

\section{Afiliación obligatoria al sistema de riesgos laborales}

La normativa colombiana (artículo 13 del Decreto 1295 de 1994) disponía lo referente a los afiliados obligatorios y voluntarios del llamado Sistema de Riesgos Profesionales, sin embargo, a partir del 11 de julio del 2012, la Ley 1562 estipuló en su artículo $2^{\circ}$ los afiliados, a saber:

a. En forma obligatoria:

1. Los trabajadores dependientes nacionales o extranjeros, vinculados mediante contrato de trabajo escrito o verbal y los servidores públicos; las personas vinculadas a través de un contrato formal de prestación de servicios con entidades o instituciones públicas o privadas, tales como contratos civiles, comerciales o administrativos, con una duración superior a un mes y con precisión de las situaciones de tiempo, modo y lugar en que se realiza dicha prestación.

2. Las Cooperativas y Precooperativas de Trabajo Asociado son responsables conforme a la ley, del proceso de afiliación y pago de los aportes de los trabajadores asociados. Para tales efectos le son aplicables todas las disposiciones legales vigentes sobre la materia para trabajadores dependientes y de igual forma le son aplicables las obligaciones en materia de salud ocupacional, incluyendo la conformación del Comité Paritario de Salud Ocupacional (COPASO). 
3. Los jubilados o pensionados, que se reincorporen a la fuerza laboral como trabajadores dependientes, vinculados mediante contrato de trabajo o como servidores públicos.

4. Los estudiantes de todos los niveles académicos de instituciones educativas públicas o privadas que deban ejecutar trabajos que signifiquen fuente de ingreso para la respectiva institución o cuyo entrenamiento o actividad formativa es requisito para la culminación de sus estudios, e involucra un riesgo ocupacional, de conformidad con la reglamentación que para el efecto se expida dentro del año siguiente a la publicación de la presente ley por parte de los Ministerio de Salud y Protección Social.

5. Los trabajadores independientes que laboren en actividades catalogadas por el Ministerio de Trabajo como de alto riesgo. El pago de esta afiliación será por cuenta del contratante.

6. Los miembros de las agremiaciones o asociaciones cuyos trabajos signifiquen I fuente de ingreso para la institución.

7. Los miembros activos del Subsistema Nacional de primera respuesta y el pago de la afiliación será a cargo del Ministerio del Interior, de conformidad con la normatividad pertinente.

En cuanto a la afiliación obligatoria de los estudiantes al Sistema de Riesgos Profesionales, hoy riesgos laborales, no es mucho lo que se ha definido, por ejemplo, no se tiene registro jurisprudencial y tampoco se ha desarrollado el artículo 2 de la Ley 1562 de 2012; en el Concepto 072 del 3 de noviembre de 2003, emitido por la Secretaría General de la Alcaldía Mayor de Bogotá, D. C., se afirma que no hay ley que reglamente la materia, y que el Gobierno Nacional, previo concepto del Sistema General de Riesgos, decidirá la oportunidad de la incorporación de los estudiantes a este seguro, escudándose así de la responsabilidad que le atañe a las entidades y universidades de afiliar los estudiantes al sistema; el profesor Alfredo Puyana Silva no desarrolla mayor contenido sobre el tema, solo lo enuncia; y el profesor Germán Arenas Monsalve (2011) afirma que esta modalidad de afiliación ha sido reglamentada respecto de los programas académicos en el área de salud, en los cuales la ley exige formalizar los llamados Convenios Docente-Asistenciales con instituciones de salud.

Si solamente existe la cobertura hacia los estudiantes del área de la salud ¿qué sucede con los demás programas académicos en los cuales los estudiantes realizan prácticas y se enfrentan a riesgos? ¿Está siendo útil la norma al ser necesaria una reglamentación para que los estudiantes se puedan ver cobijados por este derecho?

La norma estipula quiénes son afiliados obligatorios al Sistema General de Riesgos Laborales, y al igual, quiénes pueden afiliarse de manera voluntaria, y para ello se debe tener en cuenta como ya se describió el vinculo, relación o contrato, así como el nivel del riesgo, empero nuestra constitución protege el derecho al trabajo dentro del rango constitucional haciendo énfasis en que es un derecho del cual gozan todas las personas como se define en el artículo 25 de la norma de normas. En este orden de ideas el Código Sustantivo de Trabajo en su artículo $5^{\circ}$ estipula que "el trabajo es toda 


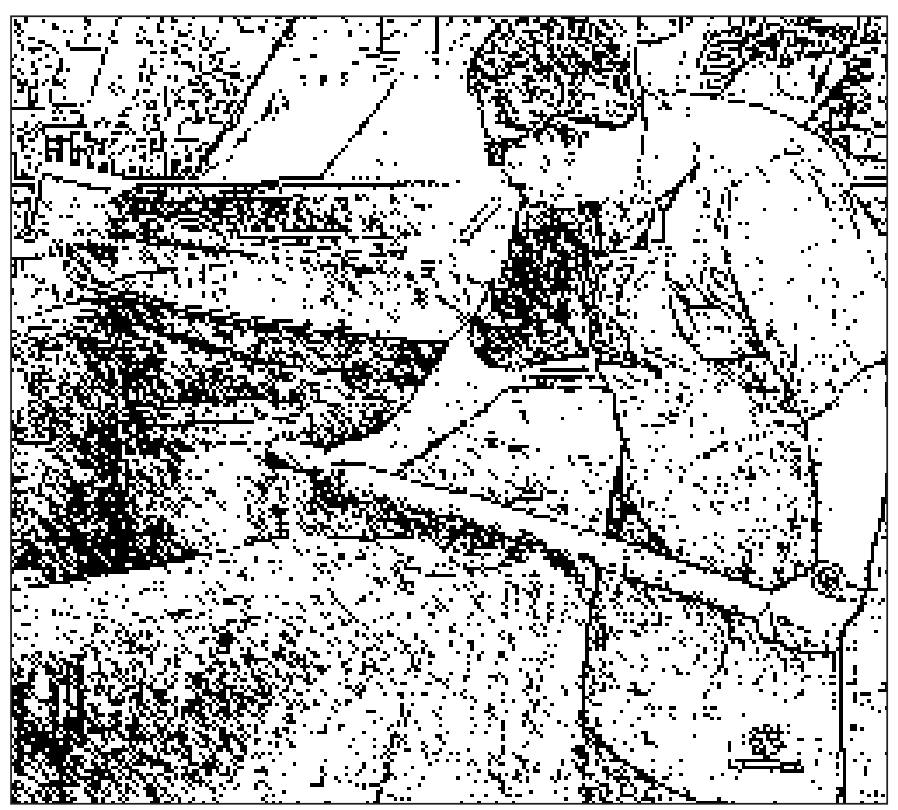

En la sociedad los riesgos son inescindibles, ya que a medida que avanza el desarrollo va generando una serie de peligros, como lo muestra el proceso que arrancó con la revolución industrial y actualmente ha supuesto un cambio radical en el modo de producción y en los procesos que este implica.

158 actividad humana libre, ya sea material o intelectual permanente o transitoria que una persona natural ejecuta conscientemente al servicio de otra y cualquiera que sea su finalidad siempre que se efectúe en ejecución de un contrato de trabajo", de esta manera para que se configure un contrato de trabajo, el Código Sustantivo del Trabajo en su artículo 23 reza que es necesario que se cumplan tres presupuestos que se deducen del mismo: a) que sea personal; b) que se pacte un salario; y, c) la continua subordinación.

Es pertinente desarrollar quiénes son trabajadores y qué se considera trabajo; Germán Rojas Gonzales $(2004,464)$ en su Diccionario de Derecho desarrolla las definiciones de dichos términos: "considera al trabajador como una persona física que presta a otra, física o moral, un trabajo personal y subordinado, entendiendo trabajo como toda actividad humana intelectual o material dirigida a la producción de cosas materiales o el cumplimiento de un servicio público o privado".

De lo anterior, se deduce que el estudiante universitario en el momento de sus prácticas, se estaría configurando los tres presupuestos, esto es, estaría en presencia de una relación laboral, ya que se reciben órdenes de la universidad y/o de la entidad en la cual se presta el servicio.

De manera clara podemos inferir que un estudiante que realiza prácticas no adquiere la calidad de trabajador, ya que está realizando un proceso de aprendizaje, que si bien ejerce la misma función de un profesional, aún no puede entenderse que está en un rol laboral; de esta manera, ya que las prácticas o pasantías son requisitos de grado para adquirir el título de profesional, no configuran 
el elemento esencial del contrato laboral (no estamos en presencia del requisito de la subordinación contractual laboral), ya que la relación es entidad-universidad, no entidad-estudiante, no se recibe remuneración por la labor que se presta, empero, aunque se acuerda un horario para una labor, no se configura el principio de contrato realidad, puesto que el objeto de desempeñar la labor es como requisito para obtener el grado profesional, no para desvirtuar la protección al trabajo.

Sin embargo, aunque no se configuran los elementos del contrato realidad, es pertinente analizar si la prestación del servicio de un estudiante a cualquier tipo de empresa, le genera una utilidad a esta última; si bien no conforma una prestación de tipo "profesional”, las funciones que muchas veces realiza el practicante o pasante son las mismas, siendo así, que se ven expuestos a riesgos laborales como cualquier trabajador.

\section{Análisis de riegos laborales en países latinoamericanos}

En los diferentes países existen varias normas que regulan el tema, cada uno de acuerdo con su soberanía, en concordancia con sus necesidades y obligaciones como Estado; en pro siempre de avanzar y en búsqueda de la felicidad del ser humano a través de un derecho representado en el deber ser.

\section{Riegos laborales en pasantes o practicantes en Argentina}

La Ley $N^{\circ} 26.427$ de Pasantías Educativas, crea el Sistema de Pasantías Educativas en el marco del sistema educativo nacional, y contempla que los estudiantes deben estar afiliados a Riesgos del Trabajo, según el ámbito de aplicación de la Ley $\mathrm{N}^{\circ} 24.557$ sobre Riesgos del Trabajo; siendo este un requisito sine qua non para firmar el Convenio de la Pasantía Educativa con alguna entidad, cabe resaltar que lo que hizo esta ley fue ampliar el régimen de cobertura, ya que la norma sobre Pasantías de Formación Profesional regulado por la Ley N ${ }^{\circ} 25013^{4}$ planteaba la obligación por parte de la empresa a otorgarle al pasante una cobertura en salud de Riesgos del Trabajo; luego el Decreto 1227 de 2001 dispuso que el empleador debía otorgar al pasante una cobertura en salud cuyas prestaciones serían las previstas en el programa médico obligatorio; anteriormente el Decreto 491 de 1997 había incorporado al régimen de esa ley a los pasantes referidos en el Decreto 340 de 1992.

4. Argentina, Congreso de la Nación. Ley No 25.013. (Septiembre 2, 1998). Establece un régimen de reforma laboral que incluye la modificación de algunos aspectos de la regulación del Contrato de Trabajo y de las Leyes $\mathrm{N}^{\circ}$. 24.013, 24.465 y 24.467, como así también de la normativa vigente en materia de convenciones colectivas de trabajo. Boletín Oficial: No 28.987, (Septiembre 22, 1998). Buenos Aires. 
Para que los estudiantes que realizan pasantías o prácticas empresariales, sean incorporados de manera efectiva en la legislación acerca de los Riesgos del Trabajo, el Estado argentino dispuso en la ley referida que los alumnos se debían incluir dentro de su ámbito como trabajadores vinculados por relaciones no laborales; que la inclusión prevista se encuentra fundamentada en los Principios de Universalidad del sistema de protección previsto en la "Ley sobre Riesgos del Trabajo" pero sin afectar el carácter no laboral de dichas vinculaciones.

Que en tal carácter resulta conveniente incluir a las personas que realizan actividades en virtud de diversas normas que establecen figuras atípicas de relaciones no laborales tales como los pasantes regulados en el Decreto 340 de 1992, los aprendices conforme al régimen de la Ley $\mathrm{N}^{\circ} 24.465$, los que desempeñan actividades en virtud del cumplimiento de una beca; y quienes prestan servicios y se capacitan en los programas especiales creados por la Ley $\mathrm{N}^{\circ} 24.013$.

Las características de todas estas actividades, en la medida que corresponda por el carácter no laboral de las prestaciones de servicios o actividades, hacen necesario poner en cabeza del empresario o dador de tareas las obligaciones que la Ley -24.013- impone a los empleadores.

160 Tal legislación se generó a raíz de las quejas presentadas por los estudiantes al ver que ellos estaban siendo explotados generando entonces un: "fraude laboral cometido por algunas empresas. Los pasantes no realizaban tareas relacionadas con sus estudios, y en algunos casos eran obligados a trabajar durante jornadas de hasta 10 y 12 horas" (www.losrecursoshumanos.com).

\section{Riegos Profesionales en pasantes o practicantes en Perú}

El 25 de mayo de 2005 entró en vigencia la Ley $\mathrm{N}^{\circ}$ 28518, sobre Modalidades Formativas Laborales que introduce una nueva regulación a las formas de capacitación o formación para el trabajo. Este marco normativo, por un lado, conlleva a que las empresas estén obligadas a pagar beneficios de naturaleza laboral a las personas contratadas bajo estos convenios, y, por otro, faculta a las empresas a contratar personal capacitado, a través de los Convenios de Prácticas Profesionales y actualización para la reinserción laboral, por el pago de beneficios sociales mínimos.

Esta regulación presenta por un lado, el otorgamiento de diversos beneficios a las personas contratadas a través de convenios formativos, lo cual se traduce, en la obligación de la empresa de asumir mayores sobrecostos laborales en caso de emplear estas modalidades de contratación; por el otro, la incorporación de nuevas modalidades contractuales, como los convenios de prácticas profesionales, pasantías y actualización para la reinserción laboral. 
Es de notar que de acuerdo a las normas anteriormente vigentes las empresas contrataban a practicantes o jóvenes capacitándolos por un determinado periodo, sin que la relación contractual generase la asunción de costos laborales ni beneficios sociales. Este escenario ha sido modificado, debido a que el legislador ha optado por otorgar nuevos beneficios a las personas que presten servicios en empresas bajo estos convenios.

En la reglamentación vigente se tiene como "aprendiz" a quien realiza prácticas pre-profesionales (entiéndase como la que se realiza durante el curso de sus estudios), y deja la calificación de "practicante" a quien realiza prácticas profesionales (aquella que se lleva a cabo al finalizar con el pensum de su carrera universitaria o técnica-profesional). Así se puede entonces analizar un poco los beneficios que tiene cada uno, por ello al ser examinada la ley 28.518 de 2005 se determina que:

a) Prácticas pre-profesionales: Es la modalidad que permite a la persona en formación durante su condición de estudiante aplicar sus conocimientos, habilidades y aptitudes, mediante el desempeño en una situación real de trabajo.

Este aprendizaje con predominio en el Centro de Formación Profesional se realiza mediante un Convenio de Aprendizaje que se celebra entre:

- Una empresa;

- Una persona en formación; y

- El tiempo de duración del convenio es proporcional a la duración de la formación y al nivel de calificación de la ocupación.

b) Práctica profesional: Es la modalidad que busca consolidar los aprendizajes adquiridos a lo largo de la formación profesional, así como ejercitar su desempeño en una situación real de trabajo.

Este aprendizaje denominado Práctica Profesional se realiza mediante un Convenio de Práctica Profesional que se celebra entre:

- Una empresa

- Una persona que egresa de un Centro de Formación Profesional o Universidad.

El tiempo de duración del convenio no es mayor a doce (12) meses, salvo que el Centro de Formación Profesional o Universidad, por reglamento o norma similar, determine una extensión mayor.

El egresado deberá ser presentado a una empresa por el Centro de Formación Profesional o Universidad, quien deberá llevar el registro del número de veces que se acoja a esta modalidad, hasta que complete el período máximo de la práctica profesional (Ministerio del trabajo del Perú). 
c) Pago de una remuneración al estudiante o egresado: aun cuando se trate de prácticas, el estudiante o egresado presta un servicio y ello tiene como contraprestación el pago de una subvención econó-

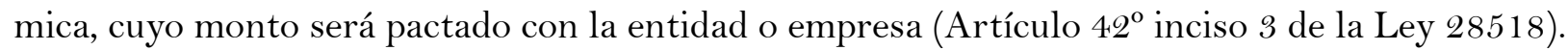
Esta subvención no puede ser menor que la Remuneración Mínima Vital si las prácticas son por la jornada máxima, y si la jornada es menor, la subvención debe ser proporcional.

d) Seguro de enfermedades y accidentes: mientras duren las prácticas debe la empresa o entidad garantizar al practicante la cobertura de enfermedades y accidentes que pudieren ocurrirle (Artículo $42^{\circ}$ inciso 7 de la Ley 28518). Esta cobertura debe ser hasta un máximo de 14 subvenciones mensuales para el caso de enfermedades y de 30 subvenciones mensuales para el caso de accidentes y dependiendo de la elección de la empresa o entidad, estará a cargo ya sea de la Entidad de Seguridad Social del Estado (ESSALUD) o de un seguro privado.

Esto debe implicar que ESSALUD establezca el costo que cobrará por esta cobertura, ya que al no constituir los beneficiarios de estos programas asegurados regulares, las empresas no están sujetas al aporte del $9 \%$. Además debe ser un monto menor por cuanto ese seguro no tiene cobertura plena, sino solo hasta 14 y 30 subvenciones mensuales conforme se ha explicado. Pero, en caso de que esas

162 empresas no cumplan con contratar alguno de los seguros indicados (ESSALUD o seguro privado), la empresa deberá “asumir directamente el costo de estas contingencias”. Esto significa que deberá asumir la totalidad del costo de la enfermedad o del accidente, lo que constituye la contingencia en materia de seguridad social. Así las empresas deben tomar las debidas precauciones sobre estos efectos cuyos costos son considerables. (AELE. Modalidades formativas: la Seguridad Social en Salud. [en línea]. Recuperado el 6 de julio de 2012 de http://www.aele.com).

\section{Riegos profesionales en pasantes o practicantes en Uruguay}

Rama Claudio (2011) reseña que antes que en el año 2000 fuese creado el Régimen de Pasantías Educativas, se tenía establecido que los estudiantes que accedieran a la educación en forma gratuita debían, a manera de contraprestación, proporcionar un servicio a la comunidad, algo similar a una práctica, pero que en el fondo estaba para llenar los vacíos en empleos que era necesario cubrir, para que el Estado cumpliera con sus obligaciones; sin embargo, este tipo de trabajo era realizado solo por estudiantes de medicina; a sabiendas, que no solo estos tienen competencias, sino que todos los profesionales cumplen un rol social y en tal sentido todos ellos debieran de realizar actividades preprofesionales como contraprestación de la gratuidad de la educación.

Así es, que el Gobierno de turno dispuso a través de la Ley 17.230 el Sistema de Pasantías Laborales, como mecanismo para regular la formación curricular de los alumnos del Subsistema de Educación 
Técnico-Profesional de la Administración Nacional de Educación Pública. También aplicable “a los alumnos inscritos en institutos privados de educación técnico-profesional que se hallen debidamente habilitados".

A través del Decreto núm. 425/001, se reglamentó el Sistema de Pasantías, creado en el año 2000, estableciendo así, que al momento en que el ente educativo firme el convenio con la empresa este debe de manera obligatoria establecer la afiliación de los estudiantes a un seguro que los cubra de accidentes o enfermedades relacionados con el trabajo que vayan a realizar, todo esto en relación a la protección sobre Riesgos Profesionales de los estudiantes.

En Uruguay la situación del estudiante frente a la empresa es mediante un contrato tripartito, en el que participan la empresa contratante, la universidad y el estudiante. Está dirigido a jóvenes de hasta 29 años de edad, y su duración no puede exceder los dos años.

La red de graduados de la Universidad ORT de Uruguay determina que: "existe un periodo de prueba, en el cual la empresa puede rescindir el contrato sin generar ningún tipo de responsabilidad. Durante el periodo de duración del contrato, el pasante será evaluado tanto por la universidad como por la empresa”.

El decreto 425 del 7 de noviembre de 2001 en su artículo 3 establece claramente que la actividad que desarrolle cada estudiante en la empresa respectiva por considerarse de naturaleza técnico-pedagógica, no será computada a los efectos jubilatorios, ni generará por sí misma derecho a permanencia o estabilidad, más allá del límite de plazo previsto en el respectivo convenio de pasantía, sin perjuicio de las causales de rescisión previstas por la ley y de las que eventualmente decida incorporar la Administración Nacional de Educación Pública.

Por ende es la compañía donde se presta el servicio, la encargada de la protección de los alumnos mientras cumplen con el horario establecido por el convenio; y no la universidad a la que esté matriculado; por eso para incentivar a las empresas a que vinculen estudiantes el gobierno uruguayo dispuso extender los beneficios para todas aquellas empresas que cumplieran a cabalidad con lo dispuesto en la norma sobre pasantías creada en el 2000, así entonces gozarán de las siguientes exoneraciones establecidas a través de la Ley 16873 (Aportes patronales con destino al régimen jubilatorio y aportes patronales con destino al seguro social por enfermedad).

Dichas exoneraciones alcanzarán a la materia gravada que generen las contrataciones celebradas en el marco de la presente ley y por el plazo de las mismas, a partir de la inscripción prevista en el artículo 31 de dicha ley. Si el empleador rescindiere unilateralmente la relación laboral antes del vencimiento del plazo, y con excepción a lo dispuesto en el artículo 30 de la presente ley, deberá 
reintegrar al Banco de Previsión Social los aportes previstos en el presente artículo. Dicho reintegro guardará correspondencia con el período por el cual se mantuvo la relación laboral.

En la actualidad por disposición de la Ley No 18.406 el Instituto Nacional de Empleo y Formación Profesional, es el encargado de vigilar y controlar el Sistema de Pasantías, para que cumpla con los objetivos que en un principio se establecieron; pero sobre todo respaldando al estudiante que realiza pasantías o prácticas empresariales.

Ahora bien, se ha insistido en aclarar que es la empresa la encargada de la afiliación a riesgos laborales; y por lo mismo la ley de Uruguay se encargó de aclarar que nunca se genera vínculo laboral entre los estudiantes y la empresa y que por tanto no tiene porqué haber estabilidad laboral, o demás beneficios que tiene para un empleado un contrato de trabajo con todo el lleno de los requisitos legales; es por eso, que no es limitante el hecho de que no se considere como trabajador a un estudiante que esté haciendo sus prácticas, para que este sea protegido contra accidentes o enfermedades relacionadas con el trabajo.

\section{Comparación de los sistemas de riesgos profesionales de los países de la región, con el sistema de riesgos laborales colombiano}

En Colombia la protección a estudiantes que realizan pasantías o prácticas empresariales está dada como ya se había dicho por el Decreto 1295 y la ley 1562 de 2012. Normas que establecen como afiliados obligatorios a todos aquellos estudiantes que realicen prácticas o pasantías; en consecuencia para los estudiantes la ley ha otorgado esta protección especial, de tal manera que, en comparación con otros países de Latinoamérica, Colombia tiene aún vacíos a nivel jurídico que debe llenar.

Hoy en día se cuenta con figuras jurídicas como el Contrato de Aprendizaje, la Asistencia Docencia, que son similares a otras que se manejan en los diversos países, además de la obligatoriedad que tienen las universidades colombianas de brindar a sus estudiantes un seguro para la protección en relación con sus estudios, esto deja en evidencia que el Estado ve la necesidad de proteger a todos aquellos que están en formación; necesidad que países como Argentina, Uruguay y Perú notaron, razón por la cual aseguran de manera efectiva a todos los alumnos; se tenían que crear entonces figuras jurídicas que se ajustaran al perfil de aseguramiento que tienen los educandos, como por ejemplo el título de "trabajadores vinculados por relaciones no laborales" o "actividad de naturaleza técnico-pedagógica”, es entonces, que en Colombia sería posible crear una figura análoga que supla el vacío y que permita que los estudiantes sean vinculados efectivamente en el Sistema de Riesgos Laborales. 


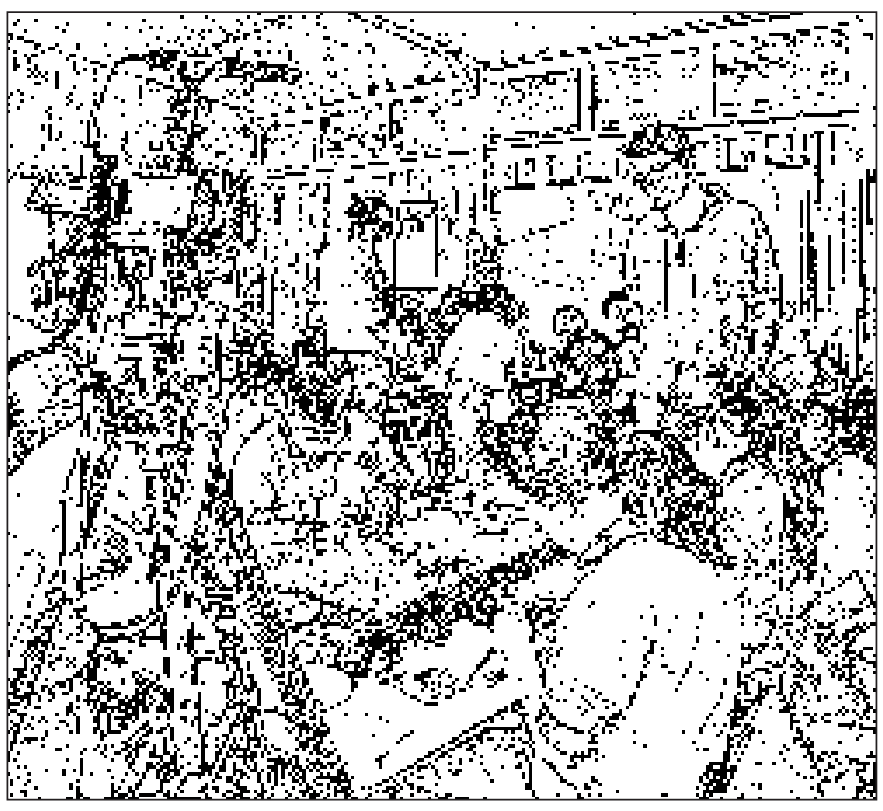

En Colombia la protección

a estudiantes que realizan pasantías o

prácticas empresariales está dada por

el Decreto 1295 y la ley 1562 de 2012.

Normas que establecen como afiliados

obligatorios a todos aquellos

estudiantes que realicen prácticas o pasantías; para los estudiantes la ley ha otorgado esta protección especial, de tal manera que, en comparación con otros países de Latinoamérica, Colombia tiene aún vacíos a nivel jurídico que debe llenar.

Aunque son evidentes las diferencias que existen entre Colombia y los demás países estudiados, también son varias las similitudes, queriendo denotar que existen a la par puntos de encuentro, $\mathrm{y}$ puntos de distanciamiento, puesto que desde el fundamento axiológico todos van dirigidos a la protección contra factores de riesgo, por ello, principios tales como la universalidad y eficiencia en el Sistema de Seguridad Social son fundamentales en las normas que regulan este tema, más aún cuando se desarrollan nuevas leyes como lo son en este caso sobre derechos de pasantes o practicantes empresariales incluido el de "la afiliación", siendo estos los mismos que encontramos en dichas legislaciones, empero, se denota un mejor tratamiento, una mayor regulación a favor de los estudiantes latinoamericanos, en donde además de una remuneración se le ampara al estudiante por la exposición a cualquier riesgo que se derive o pueda derivarse de la labor que realiza para obtener su título profesional. Así, que basándonos en esas similitudes se puede concluir que Colombia puede hallar una solución a la problemática, esto es, crear la mejor manera de proteger a los estudiantes.

No cabe duda de la necesidad de reglamentar este problema jurídico en nuestro país, no esperar a que nos pase lo mismo que los otros Estados, tener una protesta o revolución para atender los problemas y las necesidades de los pueblos; ya que como se evidenció en el curso de esta investigación esta fue una las razones que llevaron a que se le diera respuesta a la problemática.

En definitiva aunque existen vacíos jurídicos, Colombia respecto de sus estudiantes les presta protección por la labor social que cumplen, pero se denota, la falta de regulación de dicha protección. 
En nuestro país aunque se ha tratado de proteger al estudiante, ejemplo de esto es, con la aplicación por parte de las universidades del seguro estudiantil obligatorio para todo el alumnado; aún no ha sido suficiente, y mucho menos para el grupo en especial de los pasantes o practicantes empresariales, generando como ya se había dicho anteriormente una situación similar a la presentada en Argentina, y a manera de hipótesis esto podría llevarnos a una situación similar.

\section{Conclusiones y recomendaciones}

- El riesgo al que se ve expuesto un estudiante en tiempo de realización de sus prácticas empresariales y pasantías universitarias, es tan evidente como el de un trabajador, debido a que los dos sujetos se encuentran en un mismo medio laboral y por lo tanto la probabilidad de sufrir un daño en su salud (ya sea física o mental) es igual, por ende se denota el problema generado al no aplicarse de manera completa la normativa estipulada.

- Si bien el estudiante practicante o pasante no se constituye trabajador, puesto que no configuran los presupuestos del contrato laboral y porque están prestando una labor social para la culminación de sus deberes académicos, no quiere decir que no realicen las mismas operaciones de un trabajador y se vean expuestos a los mismos riesgos. Es por eso que el legislador los clasificó como afiliados obligatorios por la exposición que puede presentarse.

- En cuanto a los riegos laborales dentro de las universidades, se deduce que debido al precepto constitucional de autonomía universitaria, cada entidad de educación superior maneja y dispone de esta afiliación, a su creer y convenir.

- Se logró evidenciar que a nuestra legislación aún le hace falta mucho desarrollo en cuanto a la seguridad social y la protección a los derechos fundamentales, comparada con países de América Latina en temas de protección a estudiantes dentro del sistema de riesgos laborales.

- Es posible aplicar figuras jurídicas en Colombia como la que se crearon en países latinoamericanos como se evidenció en la investigación en derecho comparado para regular la afiliación al sistema de riesgos laborales y que los estudiantes no tengan que seguir afiliados a un seguro comercial como se ha venido realizando, puesto que aunque la normativa colombiana lo obliga, ello no hace referencia a la cobertura por el sistema de seguridad social integral.

- Con la ley 1562 del 12 de julio de 2012 que realizó la modificación al decreto 1295 de 1994, se dio un gran avance en la legislación en cuanto a la protección a estudiantes en el sistema de riesgos laborales convirtiéndolo en ley y por ende será obligación del Gobierno Nacional en cabeza de 
los Ministerios de Salud y Trabajo regularla.

- Figuras como trabajadores vinculados por relaciones no laborales o actividad de naturales técnicopedagógica pueden ser una forma de regular el tema objeto de investigación.

\section{Referentes bibliográficos}

\section{LIBROS}

Constitución Política de Colombia de 1991. Anotada. (Octubre, 2008). Editorial LEYER. (26 ed.) Bogotá, D.C.

Gonzalez Rojas, G. (2004, 2ª ed.). Diccionario de Derecho. 3R Editores. Bogotá, D.C.

FischHoff, Watson y Hope (s.f) Percepción del riesgo y de la Comunicación Revisión Anual de Salud Pública Vol. 14: 183-203 (Volumen fecha de publicación mayo de 1993)

Puyana Silva, A. (2011, $1^{\circ}$ ed.). El Sistema General de Riesgos Profesionales en Colombia. Universidad Externado de Colombia.

Puy Rodríguez, A. (1994) Percepción Social Del Riesgo Dimensiones De Evaluación Y Predicción, citando a Fischhoff, Watson y Hope, Madrid.

YATES, F. \& STOnE, E. (1992). The risk construct. En: F, Yates (Ed). Risk taking behavior. John Willey and sons, Englan, 1-25.

Ministerio de Trabajo y Seguridad Social. Conozca el Sistema General de Riesgos Profesionales. (Octubre, 1995). Pontificia Universidad Javeriana.

Ministerio de Trabajo y Seguridad Social. Código Sustantivo del Trabajo. (22 de junio, 1994). Op. cit. Artículo $23^{\circ}$.

Posada G., C. (1994, $3^{\circ}$ ed.). Los Seguros Sociales Obligatorios en España Madrid - España.

Rengifo, J. M. (1989, $3^{\circ}$ ed.). Seguridad Social en Colombia. Editorial Temis. Bogotá, D.C.

\section{NORMATIVA}

Colombia, Congreso de la República. Ley 100 (23 de diciembre, 1993). Por la cual se crea el Sistema de Seguridad Social Integral y se dictan otras disposiciones. Diario Oficial No. 41.148. Bogotá, D.C. Colombia, Congreso de la República. Ley 1562. (11 de julio, 2012). Op. cit.

Colombia, Ministerio de Trabajo y Seguridad Social. Decreto 1295. (22 de junio, 1994). Por el cual se determina la organización y administración del Sistema General de Riesgos Profesionales. Diario Oficial No. 41.405. (24 de junio de 1994). Bogotá D.C.

Colombia, Presidencia de la República. Decreto 190. (25 de enero, 1996). Por el cual se dictan normas

que reglamentan la relación docente-asistencial en el Sistema General de Seguridad Social en Salud. Diario Oficial No. 42.700. (26 de enero de 1996). Bogotá D.C.

Colombia, Congreso de la República. Ley 789. (27 de diciembre, 2002). Por la cual se dictan normas 
para apoyar el empleo y ampliar la protección social y se modifican algunos artículos del Código Sustantivo de Trabajo. Diario Oficial No 45.046. Bogotá, D.C.

Colombia, Ministerio de Trabajo y Seguridad Social. Decreto 1607. (31 de julio, 2002). Por el cual se modifica la Tabla de Clasificación de Actividades Económicas para el Sistema General de Riesgos Profesionales. Diario Oficial No. 44.892. (6 de agosto, 2002). Bogotá, D.C.

Colombia, Ministerio de Trabajo y Seguridad Social. Decreto 1295. (22 de junio, 1994). Op. cit.

Colombia, Congreso de la República. Ley 1562. (11 de julio, 2012). Op. cit.

Argentina, Congreso de la Nación. Ley No 26.427. (26 de noviembre, 2008). Crea el Sistema de Pasantías Educativas en el marco del sistema educativo nacional. Unifica las leyes y decretos de Pasantías en una sola ley. Boletín Oficial: Número 31.557, (18 de diciembre de 2008). Buenos Aires. Argentina, Congreso de la Nación. Ley 24.557. (13 de septiembre, 1995). Accidentes y Riesgos del Trabajo. Boletín Oficial: número 28.242. (3 octubre de 1995). Buenos Aires.

Argentina, Congreso de la Nación. Ley No 25.013. (Septiembre 2, 1998). Establece un régimen de reforma laboral que incluye la modificación de algunos aspectos de la regulación del Contrato de Trabajo y de las Leyes $\mathrm{N}^{\circ}$. 24.013, 24.465 y 24.467, como así también de la normativa vigente en materia de convenciones colectivas de trabajo. Boletín Oficial: $\mathrm{N}^{\circ}$ 28.987, (septiembre 22, 1998). Buenos Aires.

168 Argentina, Presidencia de la Nación. Decreto 1227. (2 de octubre, 2001). Contratos de pasantías de formación profesional. Duración, Compensación dineraria de carácter no remunerativo, Fiscalización. Boletín No 29.747 (noviembre, 2001). Buenos Aires.

Argentina, Presidencia de la Nación Decreto 491. (29 de mayo, 1997). Decreto Reglamentario de la Ley sobre Riesgos del Trabajo, incorpórense al ámbito de aplicación y al sistema creado por la Ley $\mathrm{N}^{\circ} 24.557$ a los trabajadores domésticos, a los vinculados por relaciones no laborales y a los trabajadores autónomos. Boletín No 28.661 (4 de junio, 1997). Buenos Aires.

Argentina, Presidencia de la Nación. Decreto 340. (24 de febrero, 1992). Que crea el Sistema de Pasantías Boletín No 28.661 (28 de febrero, 1992). Buenos Aires.

Argentina, Congreso de la Nación. Ley N²4.465. (15 de marzo, 1995) Régimen de Contrato de Trabajo, modificación: modalidad especial de fomento del empleo, Contrato de Aprendizaje. Boletín $\mathrm{N}^{\circ} 28.112$ (23 de marzo, 1995). Buenos Aires.

Argentina, Congreso de la Nación. Ley N 24.013. (13 de noviembre, 1991). Ley nacional de empleo. Boletín No 27.286 (05 de diciembre, 1991). Buenos Aires.

Argentina, Congreso de la Nación. Ley N²4.013. (13 de noviembre, 1991). Op. cit.

Perú, Congreso de la República. Ley N²8.518. (24 de mayo, 2005). Ley sobre Modalidades Formativas Laborales. Diario Oficial $\mathrm{N}^{\circ}$ 9226. (19 de septiembre, 2005). Lima.

Perú, Congreso de la República. Ley No 28.518. (24 de mayo, 2005). Op. cit. Artículo 12. Perú, Congreso de la República. Ley No 28.518. (24 de mayo, 2005). Op. cit. Artículo 13.

Uruguay, El Senado y la Cámara de Representantes de la República Oriental. Ley 17.230. (7 de enero, 2000). Régimen de Pasantías Laborales para alumnos mayores de 15 años de Institutos Públicos 
y Privados. Diario Oficial No 25447 (24 de enero, 2000). Montevideo.

Uruguay, Presidencia de la República. Decreto No 425 (7 de noviembre, 2001). Régimen de Pasantías

Laborales para alumnos mayores de 15 años de Institutos Públicos y Privados. Promulgación: (31 de octubre, 2001). Montevideo.

ORT. Sitio web de los graduados de la Universidad ORT de Uruguay. Pasantías. Ley de Empleo Juvenil. Recuperado el 7 de julio de 2012 de http://graduados.ort.edu.uy.

Uruguay, Presidencia de la República. Decreto No 425 ( 7 de noviembre, 2001). Op. cit. Artículo $3^{\circ}$. Uruguay, El Senado y la Cámara de Representantes de la República Oriental. Ley No 16.873 (3 de octubre, 1997). Establece requisitos y otorga beneficios a empresas que incorporen a jóvenes en las modalidades contractuales. Diario Oficial No 24890 (16 de octubre, 1997). Montevideo.

Uruguay, El Senado y la Cámara de Representantes de la República Oriental. Ley No 18.406. (24 de octubre, 2008). Se crea como persona jurídica de derecho público no estatal el Instituto Nacional de Empleo y Formación Profesional. Diario Oficial: No 27608 (10 de noviembre, 2008). Montevideo.

\section{FUENTES WEB}

Corporación Ambiental Empresarial. (8 de mayo, 2006). Panorama De Riesgos. en línea]. Recuperado el 20 de julio de 2012 de http://www.corporacionambientalempresarial.org.co/documentos/ acar_panorama_riesgos_tx.pdf.

Salud Ocupacional. (5 de mayo de 2011). Cartilla 2 de Salud Ocupacional. [en línea]. Recuperado el 20 de julio de 2012 de http://salud-ocupacional-nusefa-cartilla2.blogspot.com

Colombia, Secretaría General Alcaldía Mayor de Bogotá, D.C. Concepto 072. (noviembre 03, 2003). Radicación 2-2009-59508. Recuperado el 23 de mayo de 2012 de http://www.alcaldiabogota.gov. co/ sisjur/normas/Norma1.jsp?i=38711

AELE. Análisis Tributario y Laboral. (26 de septiembre, 2011). Modalidades Formativas: La Seguridad Social en Salud. [en línea] Recuperado el 6 de julio de 2012 de http://www.aele.com/node/5456. SCRIB. Rama, Claudio. (10 de octubre, 2011). Pasantías Estudiantiles; Trabajo Académico o Empleo Encubierto. [en línea]. Recuperado el 6 de julio de 2012 de http://es.scribd.com/doc/67995779/ Las-pasantias-estudiantiles-Trabajo-academico-o-empleo-encubierto.

España, Ministerio de trabajo e Inmigración. (Septiembre, 2010) Actualidad Internacional Sociolaboral. Recuperado el 1 de agosto de 2012 de http://www.empleo.gob.es/es/mundo/Revista/ Revista138/Revista 138.pdf

\section{SENTENCIAS}

Colombia, Corte Constitucional. Sentencia T - 935. (8 de noviembre, 2007). M.P. Dr. Marco Gerardo Monroy Cabra. Expediente T-1632464. Bogotá. D.C. 